Pleione 12(1): 18 - 30. 2018.

(C) East Himalayan Society for Spermatophyte Taxonomy

ISSN: 0973-9467

doi:10.26679/Pleione.12.1.2018.018-030

\title{
Assessment of genetic diversity in cultivated Indian tea (Camellia sinensis (L.) Kuntze)
}

\author{
Manprit Bajwa, Balwinder S. Bajwa and Arnab Sen ${ }^{1}$ \\ Molecular Genetics Laboratory, Department of Botany, University of North Bengal, Siliguri 734013, \\ West Bengal, India \\ ${ }^{1}$ Corresponding author: e-mail: senarnab_nbu@hotmail.com
}

[Received 13.04.2018; Revised 20.06.2018; Accepted 21.06..2018; Published 30.06.2018]

\begin{abstract}
Tea is an important beverage crop in India and worldwide. The tea germplasm in India includes three different types of tea cultivars, namely, China type, Assam type and Cambod type. Present study was conducted to evaluate the degree and organization of genetic diversity in cultivated Indian tea. The technique used was based on random amplified polymorphic DNAPCR (RAPD-PCR) markers analysis. 34 ten decamer primers were used generating 359 reproducible bands and among these, $321(89.42 \%)$ bands were polymorphic. The genetic diversity among cultivars as calculated from Nei's index averaged 0.28 varying from 0.07 to 0.50 . Calculation of Shannon index of diversity revealed an average of 0.44 varying from 0.28 to 0.56 , indicating that Camellia sinensis (L.) Kuntze maintains a relatively high degree of genetic diversity at the cultivar level. Analysis of genetic dissimilarity showed that genetic differentiation occurred among studied cultivars, which supports the possibility of current ex-situ conservation strategies. Dendrograms were generated using UPGMA method revealing three distinct clusters of Cambod, Assam and China type, which is in agreement with the known taxonomical classification of tea. A study of correspondence analysis of the data also revealed a clear picture of polymorphism detected among the said cultivars. These results suggest that the RAPD-PCR method is a rapid and effective means to evaluate the genetic diversity among a large number of tea cultivars and help devise sampling strategies to compliment classical morpho-agronomic descriptors and conservation of Indian tea cultivars.
\end{abstract}

Key words: Camellia sinensis, tea, RAPD-PCR, Genetic diversity, Nei's diversity index, Shannon's information index

\section{INTRODUCTION}

Tea [(Camellia sinensis (L.) Kuntze], an important beverage crop, contributes significantly to the economy of many Asian countries including India (Kaundan \& Park 2002). Generally it is categorized into two main varieties: var. sinensis which originated from mainland China recognized by its small leaf, slower growing bush withstanding colder climates (Wight 1962; Banerjee 1992) and var. assamica discovered in the nineteenth century in the Assam region in India and later in other parts of south east Asia (Kaundan \& Park 2002) which can be easily identified by its large leaf, tall and quick growing characters which is well suited to very warm tropical climates (Wight 1962; Banerjee 1992). Besides these two basic varieties, there exists Camellia assamica var. lasiocalyx (G. Watt) A.P. Das \& C. Ghosh (Das \& Ghosh 2016) with its intermediate leaf size that is generally referred as cambod variety. The assamica variety contains large amount of tannin and catechin and is particularly used for preparing black tea, whereas sinensis tea accounts for most of the green tea production 
(Kaundan \& Matsumoto 2003). Tea being an out crossing crop, a large variation in several important and desirable characters occurs from bush to bush in the existing tea populations (Ghosh 2001). Selection and breeding for the production of desirable traits in this perennial crop have resulted in severe erosion of its genetic base over the time. Lack of proper conservation programmes have caused major reduction in its gene pool, as most of the breeding and conservation programs are still based on conventional morphological and agronomical descriptors, which are dependent on environmental and developmental factors thus reflecting the base of the gene pool (Green 1971; Wilkremaratne 1981) with no true genetic relatedness. In order to stop further reduction in its gene pool and to breed for new tea types with more productiveness; less prone to natural calamities, diseases, as well as new flavors, a thorough knowledge of the existing genetic diversity is a pre-requisite.

Recently, a large number of DNA markers are in use as tools for large scale genetic studies providing the best estimate of genetic diversity and are not influenced by the environmental factors. Various classes of the molecular markers are available but those based on PCR are considered to meet many applications in genetic studies (Powell et al. 1995). Although techniques like RFLP and AFLP have been applied successfully and have provided considerable genetic information in a number of plant species (Zhang et al. 1992; Vos et al. 1995; Xu et al. 2000), these techniques are comparatively slow and expensive requiring the nucleotide information and are not amenable for assessment of genetic studies. More recently PCR based RAPD and SSR markers have been developed (Williams et al. 1990; De Bustos et al. 1998). SSR markers require prior information of target DNA sequences and sophisticated electrophoretic systems with computer software for accurate band separation and scoring (Smulders et al. 1997; Sun et al. 1998). RAPD techniques overcome these limitations; a number of polymorphic markers can be obtained with ease from small amount of genomic DNA without the knowledge of target sequences. There is only one drawback of this technique i.e., lack of reproducibility that may arise if the experimental conditions applied are not standardized properly (Prenner et al. 1993). In spite of this fact, RAPD methodology provides informative data consistent with other markers, especially at the intraspecific level (Dos Santos et al. 1994; Lerceteau et al. 1997). Moreover it is cost effective for large scale population genetic analysis. In preliminary studies, the importance of molecular markers for the characterization of tea genetic resources and estimation of genetic diversity were reported by various workers (Wachira et al. 1995; Paul et al. 1997; Kaundan \& Park 2002). Bera and Saikia (1999) have screened five varieties of tea from north east region using sixteen RAPD markers but the work mainly covered only the molecular characterization and genetic diversity. Therefore, present study is a further attempt to rationalize tea cultivars by employing RAPD methodology to determine the level and distribution of genetic diversity in twenty six tea populations using 34 ten mer primers.

\section{MATERIAL AND METHODS}

\section{Plant Material}

Twenty six different varieties of tea were collected from different regions of North Bengal (Table 1) and maintained as germplasm in the tea garden of Noth Bengal University, West Bengal.

\section{DNA extraction}

Total genomic DNA was extracted by CTAB method (Dellaporta et al. 1983) with slight modifications. Tender leaves two and a bud shoots were taken weighing approx. five grams 
Table 1. Description of tea cultivars used in the present study. Abbreviations: TV-Tocklai Vegetative, HV-Happy Valley, TSS-Tocklai Seed Stock, BS-Balasun, K-Kopati, PPhoobshering, B-Bannockburn, RR-Runglee Rungliot, T-Tukdah, CP-Clonal Proving, AVAmbari Vegetative

\begin{tabular}{|c|c|c|c|c|}
\hline S1.No. & Clone & Species & Type & Origin \\
\hline 1 & TV29 & C.assamica sub.Lasiocalyx & Cambod & Tocklai ExperimentalStation \\
\hline 2 & TV17 & C.assamica & Assam. & Tocklai ExperimentalStation \\
\hline 3 & TV22 & C.assamica sub.Lasiocalyx & Cambod & Tocklai ExperimentalStation \\
\hline 4 & HV39 & C.sinensis (L.)O.Kuntze & China & Darjeeling \\
\hline 5 & TV20 & C.assamica sub.Lasiocalyx & Cambod & Tocklai ExperimentalStation \\
\hline 6 & TS449 & Seed stock & Seedstock & Tocklai ExperimentalStation \\
\hline 7 & BS71A76 & C.sinensis (L.)O.Kuntze & China & Darjeeling \\
\hline 8 & $\mathrm{~K} 1 / 1$ & C.assamica & Assam & Darjeeling \\
\hline 9 & TV30 & C.assamica sub.Lasiocalyx & Cambod & Tocklai ExperimentalStation \\
\hline 10 & P1258 & C.sinensis (L.)O.Kuntze & China & Darjeeling \\
\hline 11 & TV27 & C.assamica sub.Lasiocalyx & Cambod & Tocklai ExperimentalStation \\
\hline 12 & TV1 & C.assamica & Assam/China & Tocklai ExperimentalStation \\
\hline 13 & B777 & C.sinensis (L.)O.Kuntze & China & Darjeeling \\
\hline 14 & RR17/144 & C.sinensis (L.)O.Kuntze & China & Darjeeling \\
\hline 15 & $\mathrm{~T} 145$ & C.assamica & Assam & Darjeeling \\
\hline 16 & $\mathrm{~T}-78$ & C.sinensis (L.)O.Kuntze & China & Takdah T.E., Darjeeling \\
\hline 17 & TV25 & C.assamica sub.Lasiocalyx & Cambod & Tocklai ExperimentalStation \\
\hline 18 & CPI & C.assamica & Assam & Darjeeling \\
\hline 19 & TV18 & C.assamica sub.Lasiocalyx & Cambod & Tocklai ExperimentalStation \\
\hline 20 & TV28 & C.assamica sub.Lasiocalyx & Cambod & Tocklai ExperimentalStation \\
\hline 21 & TV19 & C.assamica & Cambod & Tocklai ExperimentalStation \\
\hline 22 & TV23 & C.assamica sub.Lasiocalyx & Cambod & Tocklai ExperimentalStation \\
\hline 23 & TV26 & C.assamica sub.Lasiocalyx & Cambod & Tocklai ExperimentalStation \\
\hline 24 & AV2 & C.sinensis (L.)O.Kuntze & China & Darjeeling \\
\hline 25 & P312 & C.sinensis (L.)O.Kuntze & China & Darjeeling \\
\hline 26 & B157 & C.sinensis (L.)O.Kuntze & China & Darjeeling \\
\hline
\end{tabular}

in a mortar and pestle and ground into a fine powder with the help of liquid nitrogen. The pulverized material was taken in an Oakridge tube containing $15 \mathrm{ml}$ of pre-warmed $\left(65^{\circ} \mathrm{C}\right)$ $\mathrm{CTAB}$ extraction buffer. The tube was then vortexed for 5 seconds and incubated in a water bath for $1 \mathrm{hr}$ at $65^{\circ} \mathrm{C}$. Following the $1 \mathrm{hr}$ incubation an equal volume of chloroform/isoamyl alcohol (24:1) was added and the mixture was gently mixed by inverting the tube upside down. The extract was centrifuged for 10 minutes at about 10,000 rpm (room temperature) and the supernatant was carefully transferred to a fresh tube. The chloroform/Isoamyl alcohol step was repeated twice and 0.6 volume of ice cold isopropanol was added to the final supernatant. Upon gentle swirling the DNA CTAB complex precipitated as a whitish network and was spooled out of the solution using a bent Pasteur pipette. It was then washed thrice in a solution of $70 \%$ and $10 \mathrm{mM}$ ammonium acetate, dried and dissolved in $1 \mathrm{ml}$ of $1 \mathrm{X} \mathrm{TE}$ buffer ( $\mathrm{pH}$ 7.4). The dissolved DNA was extracted with an equal volume of equilibrated phenol ( $\mathrm{pH} \mathrm{8.0)}$ and then with chloroform/ isoamyl alcohol (24:1). To the upper aqueous phase 0.1 volume of $7.5 \mathrm{M}$ ammonium acetate and 2 volume of absolute alcohol was added and precipitated at $4^{\circ} \mathrm{C}$ for 30 minutes in a cooling centrifuge at $12000 \mathrm{rpm}$. The pellet obtained was washed with $70 \%$ alcohol, dried with vacuum pump and dissolved in $500 \mu 1$ of 1X TE buffer ( $\mathrm{pH} 7.4)$. 
Purification of DNA

Fifty $\mu \mathrm{g} / \mathrm{ml}$ of RNase A was added to the genomic DNA of tea dissolved in $500 \mu 1$ of $1 \mathrm{X}$ TE buffer ( $\mathrm{pH} \mathrm{7.4)} \mathrm{and} \mathrm{it} \mathrm{was} \mathrm{incubated} \mathrm{at} 37^{\circ} \mathrm{C}$ for $1 \mathrm{hr}$ in dry water bath. It was then purified with chloroform/isoamyl alcohol (24:1) and again precipitated with 2 volumes of absolute alcohol and 0.1 volume of $3 \mathrm{M}$ sodium acetate ( $\mathrm{pH}$ 5.2). The DNA pellet was finally dissolved in $500 \mu 1$ of 1 XTE ( $\mathrm{pH} 7.4$ ) buffer. DNA concentration was estimated by $0.8 \%$ agarose gel stained with ethidium bromide.

\section{RAPD analysis}

A total of 34 random 10 mer primers (Table 2) were screened belonging to KitA and KitB of Operon technologies Inc. for 26 varieties.

Table 2. Genetic polymorphism of tea cultivars detected by RAPD markers

\begin{tabular}{|c|c|c|c|}
\hline Sequences & $\begin{array}{l}\text { Bands } \\
\text { Generated }\end{array}$ & $\begin{array}{l}\text { Polymorphic } \\
\text { Bands }\end{array}$ & $\begin{array}{l}\text { \%age of } \\
\text { polymorphism }\end{array}$ \\
\hline OPA01 (CAGGCCCTTC) & 11 & 9 & 81.8 \\
\hline OPA02 (TGCCGAGCTG) & 10 & 8 & 80 \\
\hline OPA03 (AGTCAGCCAC) & 11 & 10 & 90.9 \\
\hline OPA04 (AATCGGGCTG) & 16 & 14 & 87.5 \\
\hline OPA05 (AGGGGTCTTG) & 9 & 8 & 88.88 \\
\hline OPA06 (GGTCCCTGAC) & \multicolumn{2}{|c|}{ NO AMPLIFICATION } & \\
\hline OPA07 (GAAACGGGTG) & 14 & 13 & 92.8 \\
\hline OPA08 (GTGACGTAGG) & 17 & 15 & 88.2 \\
\hline OPA09 (GGGTAACGCC) & 10 & 9 & 90 \\
\hline OPA10 (GTGATCGCAG) & 12 & 12 & 100 \\
\hline OPA11 (CAATCGCCGT) & 8 & 7 & 87.5 \\
\hline OPA12 (TCGGCGATAG) & 15 & 14 & 93.3 \\
\hline OPA13 (CAGCACCCAC) & 12 & 9 & 75 \\
\hline OPA14 (TCTGTGCTGG) & 10 & 9 & 90 \\
\hline OPB01 (GTTTCGCTCC) & 16 & 15 & 93.75 \\
\hline OPB02 (TGATCCCTGG) & 8 & 7 & 87.5 \\
\hline OPB03 (CATCCCCCTG) & \multicolumn{2}{|c|}{ NO AMPLIFICATION } & \\
\hline OPB04 (GGACTGGAGT) & 7 & 7 & 100 \\
\hline OPB05 (TGCGCCCTTC) & 12 & 12 & 100 \\
\hline OPB06 (TGCTCTGCCC) & 10 & 8 & 80 \\
\hline OPB07 (GGTGACGCAG) & 16 & 12 & 75 \\
\hline OPB08 (GTCCACACGG) & 17 & 14 & 82.3 \\
\hline OPB09 (TGGGGGACTC) & 7 & 7 & 100 \\
\hline OPB10 (CTGCTGGGAC) & 8 & 6 & 75 \\
\hline OPB11 (GTAGACCCGT) & 5 & 4 & 80 \\
\hline OPB12 (CCTTGACGCA) & 9 & 9 & 100 \\
\hline OPB13 (TTCCCCCGCT) & 15 & 15 & 100 \\
\hline OPB14 (TCCGCTCTGG) & 9 & 8 & 88.88 \\
\hline OPB15 (GGAGGGTGTT) & 10 & 8 & 80 \\
\hline OPB16 (TTTGCCCGGA) & \multicolumn{2}{|c|}{ NO AMPLIFICATION } & \\
\hline OPB17 (AGGGAACGAG) & 12 & 11 & 91.6 \\
\hline OPB18 (CCACAGCAGT) & 16 & 15 & 93.75 \\
\hline OPB19 (ACCCCCGAAG) & 12 & 11 & 91.6 \\
\hline OPB20 (GGACCCTTAC) & 15 & 14 & 93.3 \\
\hline TOTAL & 359 & 321 & 89.4 \\
\hline
\end{tabular}

PCR reactions were carried out in a $25 \mu 1$ reaction mix containing approx $25 \mathrm{ng}$ template DNA, $200 \mu \mathrm{M}$ of each dATP, dCTP, dGTP, dTTP (Sigma), $0.5 \mu \mathrm{M}$ of a single 10 base primer, 1 unit of Taq DNA polymerase (Sigma). The 1 X PCR buffer (100mM Tris HCl, pH 8.3, 500mM $\mathrm{KCl}, 15 \mathrm{mM} \mathrm{MgCl}_{2}$ and $0.01 \%$ gelatin) used was the one provided by the manufacturer of the 
enzyme. The PCR reactions were run in a thermal cycler (Applied Biosystems). The amplification cycle consisted of an initial denaturation at $92^{\circ} \mathrm{C}$, followed by 40 cycles of $1 \mathrm{~min}$. denaturation at $92^{\circ} \mathrm{C}, 1 \mathrm{~min}$. annealing at $37^{\circ} \mathrm{C}$ and $2 \mathrm{~min}$. extension at $72^{\circ} \mathrm{C}$, with a final extension at $72^{\circ} \mathrm{C}$. The PCR products were separated on $1.5 \%(\mathrm{~W} / \mathrm{V})$ agarose gel run in $1 \mathrm{X}$ TBE buffer and stained with ethidium bromide. The gels were visualized with a UV Transilluminator and photographed with GDS. A DNA ladder (lambda DNA HindIII digest) was used as a molecular size marker. All PCR reactions were run at least thrice and only reproducible and clear bands were scored.

\section{Data analysis}

Each polymorphic band was regarded as a binary character and was scored as 1 (presence) or 0 (absence) for each sample and assembled in a data matrix. A similarity matrix on the basis of band sharing was calculated from the binary data using Dice coefficient (Nei \& $\mathrm{Li}$, 1979). Similarities were graphically expressed using the group average agglomerative clustering to generate dendrograms. The analysis was done using the software package NTSYS-pc (version 2.0) (Rohlf 1998)

The POPGENE freeware (Yeh et al. 1997) was used to partition genetic diversity among the twenty six cultivars. The same software was used to calculate Nei's genetic index, genetic distances, and Shannon's information index among the tea cultivars and to draw a dendrogram based on Nei's (Nei 1972) genetic distances using the UPGMA.

Correspondence analysis was performed to graphically summarize associations among the 26 varieties. Analysis was done through a batch file following the software package NTSYS-pc.

\section{RESULTS}

Thirty-four oligonucleotide primers were used to determine genetic divergence among 26 varieties of tea. A total of 359 major scorable fragments ranging from $500-3000 \mathrm{bp}$ was generated from 31 primers as the other 3 primers could not amplify any of the samples. Out of the 359 scorable bands 321 were polymorphic. Each primer was tried thrice and the results were reproducible. The number of polymorphic bands per primer ranged from four in OPB-11 to fifteen in OPA08, OPB-01, OPB-13 and OPB-18, with an average of 10.3 polymorphic bands per primer (Table 2). Figure1 shows the RAPD-PCR amplified by primer OPA-04. These clear, repeatable and polymorphic bands were scored and used in further statistical analysis

The percentage of polymorphic bands ranged from $75 \%$ to $100 \%$. OPA- 10 , OPB- 04 , OPB-05, OPB-09, OPB-12 and OPB-13 revealed 100\% polymorphism while, OPA-13, OPB07 , and OPB-10 generated $75 \%$ polymorphism. An intermediate polymorphism $(87.5 \%)$ was revealed by OPA-04, OPA-11 and OPB-02 (Table 2).

Diversity measures were calculated by Nei's (Nei 1972) index and ranged from $\mathrm{h}=$ 0.07 to $\mathrm{h}=0.50$ (Figure 2). Averaged over all markers, primer OPB-14 showed least diversity (0.1920) among the populations and primers OPB-04 revealed the highest diversity $(0.3850)$ among the tea populations. An intermediate diversity is revealed by primers OPB- $08(0.2961)$ and OPB-17 (0.2823) at few loci for all the populations. The mean genetic diversity index (h) was 0.2893 (Table 3).

The mean value for the total diversity $(\mathrm{Ht})$ among all populations was calculated to be 0.2893. The mean level of genetic differentiation (Gst) among populations over all loci was 1.00 (Table 3 ). This indicates that $100 \%$ polymorphism is observed among populations. 


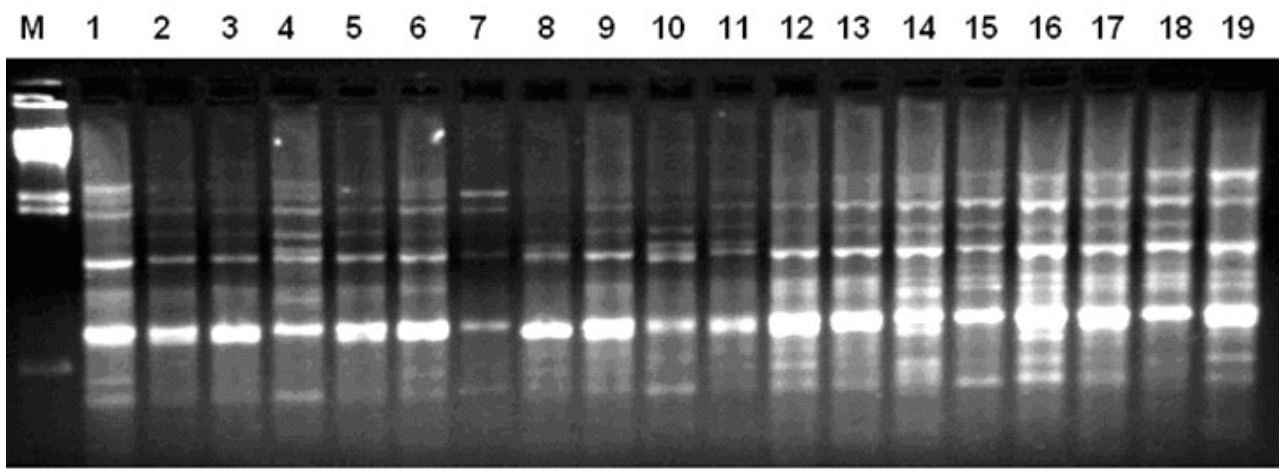

Figure 1. Amplification of different tea cultivars using RAPD primer OPA-04. Lane M shows molecular marker (lambda DNA HindIII digest). Lane $1=\mathrm{TV} 29,2=\mathrm{TV} 17,3=\mathrm{TV} 22$, 4=HV39, 5=TV20, $6=\mathrm{TSS} 449$, 7=BS71A76, 8=K-1/1, 9=TV30, 10=P1258, 11=TV27, 12=TV1, 13=B777, 14=RR17/144, 15=T145, 16=T78, 17=TV25, 18=CP1, 19=TV18. Abbreviations: TV-Tocklai Vegetative, HV-Happy Valley, TSS-Tocklai Seed Stock, BS-Balasun, K-Kopati, P-Phoobshering, B-Bannockburn, RR-Runglee Rungliot, T-Tukdah, CP-Clonal Proving, AV-Ambari Vegetative.

Table 3. Nei's analysis of gene diversity in multi-populations as a whole

\begin{tabular}{|l|l|l|l|l|}
\hline $\begin{array}{l}\text { Sample } \\
\text { size }\end{array}$ & $\begin{array}{l}\text { Nei's gene } \\
\text { diversity } \\
\text { (h) }\end{array}$ & $\begin{array}{l}\text { Shannon's } \\
\text { Information index (I) }\end{array}$ & $\begin{array}{l}\text { Total gene } \\
\text { diversity (Ht) }\end{array}$ & $\begin{array}{l}\text { Coefficient of gene } \\
\text { differentiation (Gst) }\end{array}$ \\
\hline 26 & 0.2893 & 0.4394 & 0.2893 & 1.00 \\
\hline
\end{tabular}

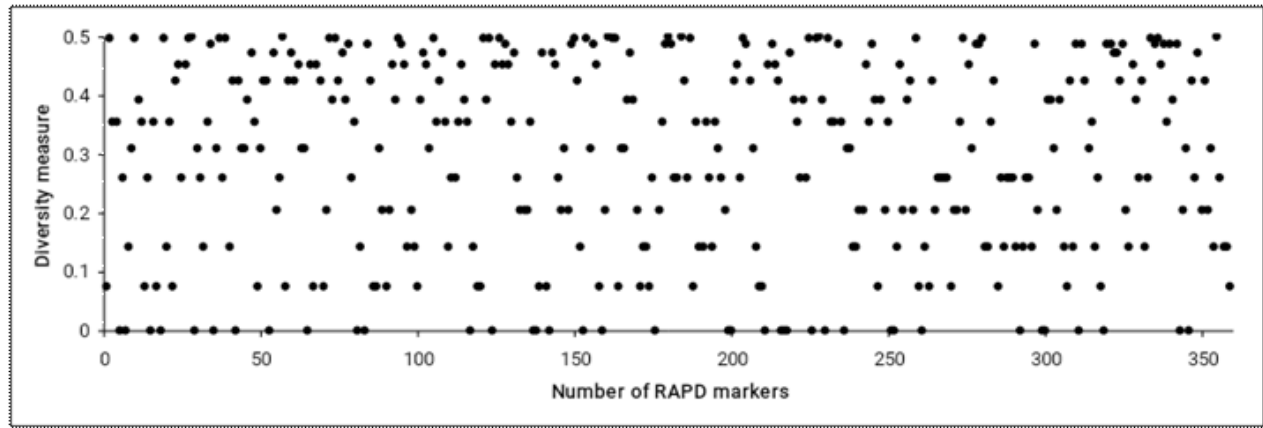

Figure 2. The figure shows genetic diversity among tea cultivars estimated by Nei's (1972) diversity measure for 359 RAPD markers.

Genetic distances calculated from the Nei's original measure of genetic distance (Nei, 1972) averaged 0.28 and varied from 0.16 , lowest between cultivars TV17 and TV22 and 0.52 , highest between the cultivars BS71A76 and TV23 (Table 4).

A dendrogram constructed on the basis of Nei's genetic distances and the UPGMA method (Figure 3) showed three main clusters: Cluster I, cluster II. and cluster III. The clusters revealed the level of relatedness between the 26 cultivars studied (Table 1). Measure of similarity ranged from a minimum $46 \%$ between TV17, TV22 and P312 to a maximum $100 \%$ between TV28 and TV23. Cluster I consist of thirteen cultivars which is the largest cluster comprising of Assam type which are divided into two main groups: The first group comprises of seven cultivars TV29, BS71A76, TV17, TV22, TV20, HV39, TSS449 and 


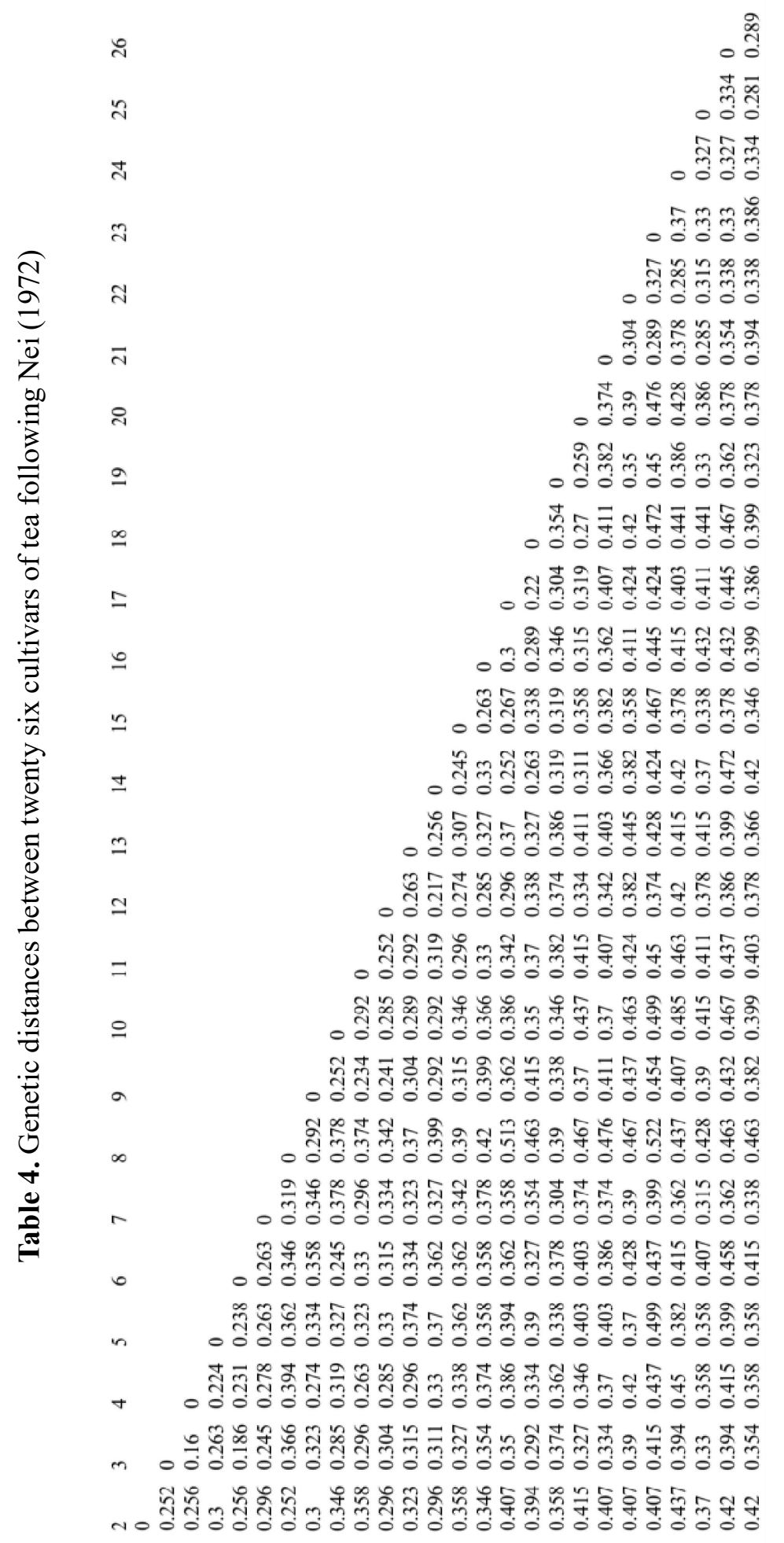

ーー 


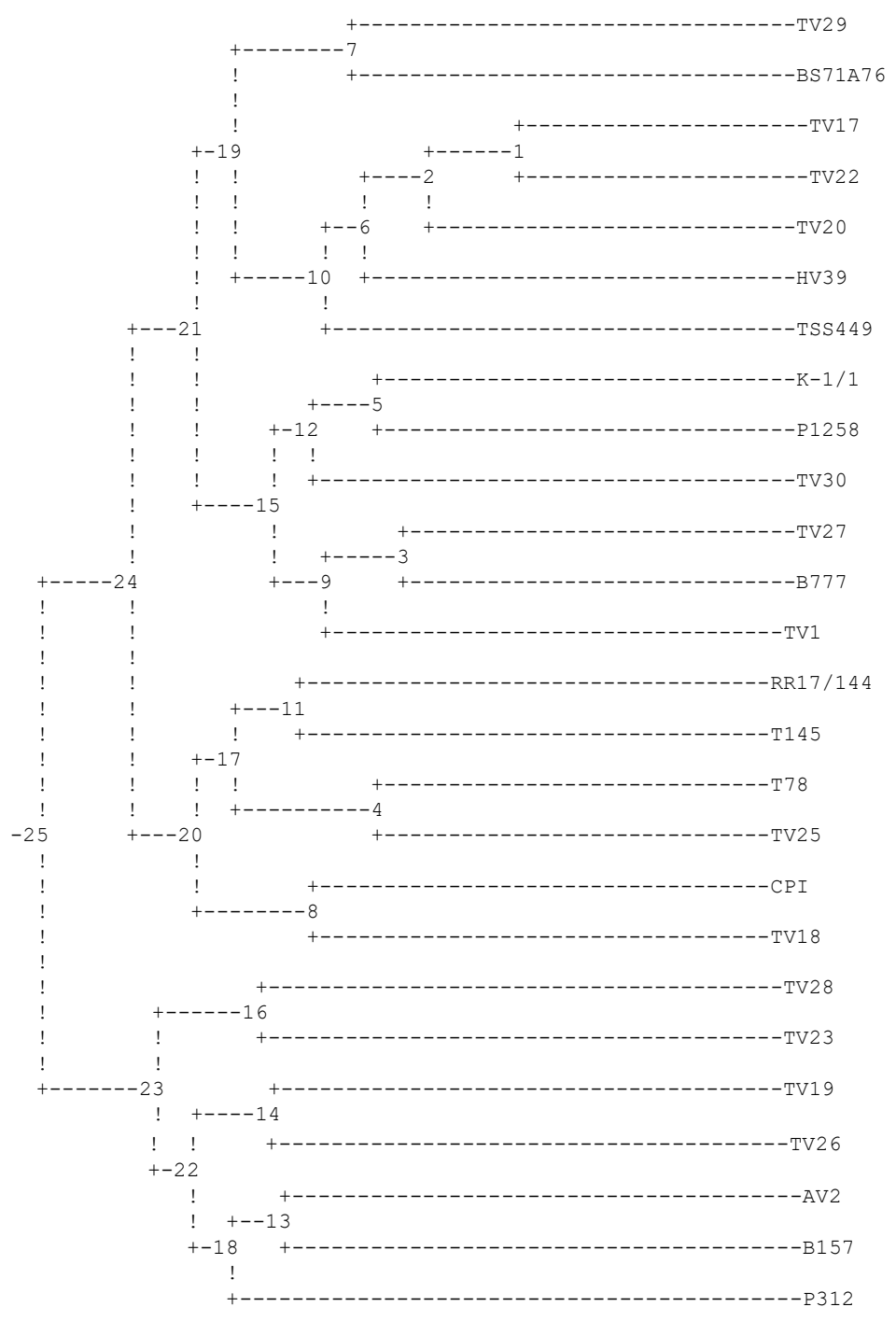

Figure 3. Dendrogram based on Nei's (1972) Genetic distance: Method = UPGMA Modified from NEIGHBOR procedure of PHYLIP Version 3.5

within this group there are two subgroups. In the first subgroup two completely different cultivars TV-29 (Assam) and BS71A76 (China) revealed height similarity of 100\% which does not match with the existing knowledge on the systematic of tea $(C$. sinensis $)$.The reason for their clustering is not presently known but possible explanation may be that traditionally tea is classified on the basis of morphological features which are subjected to substantial environmental changes coupled with extensive hybridization. The other two cultivars TV17 and TV22, although one Assam and other Cambod respectively, also revealed $100 \%$ similarity. This may be due to the reason that traditionally cambod is a subgroup of Assam type or as it is sometimes referred to as a subspecies of assamica (var. lasiocalyx) (Wight 1962). The other three cultivars, TV20 (Cambod), HV39 (China) and TSS449 (Seed stock) formed subgroups of their own. Second group comprises of six cultivars: Kopati-1/1 (Assam type), P1258 (China type), TV30 (Assam type), TV27 (Cambod), B777 (China type), TV1 


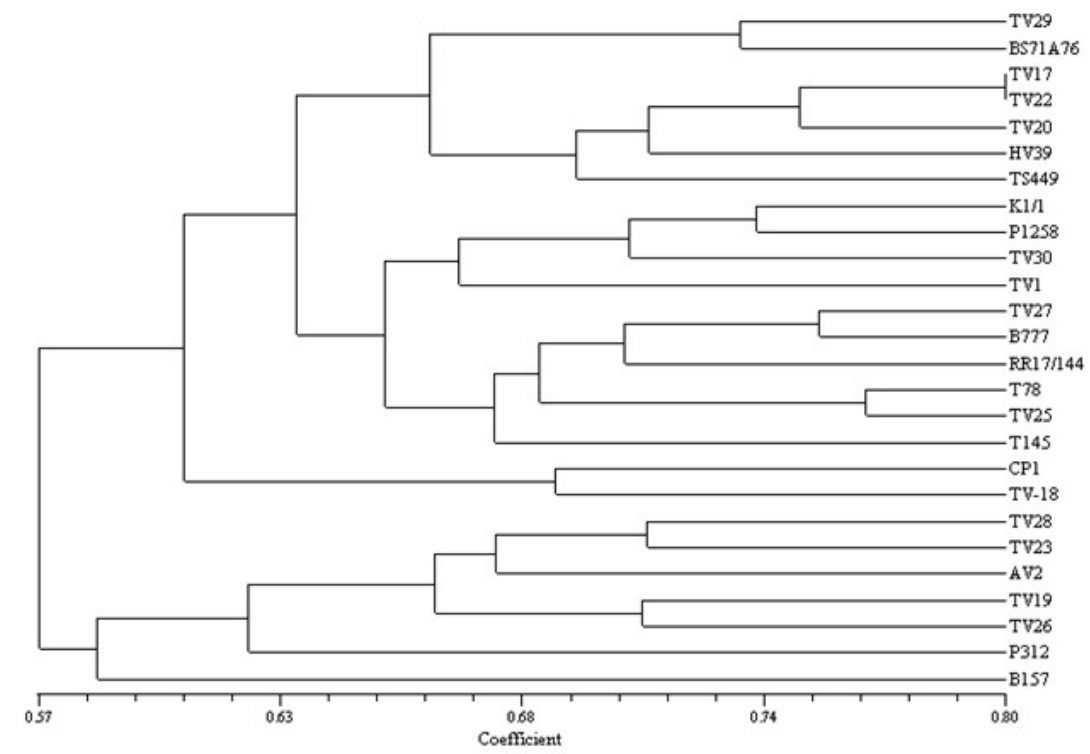

Figure 4. Dendrogram based on NTSYS illustrating the relationships among twenty six tea cultivars.

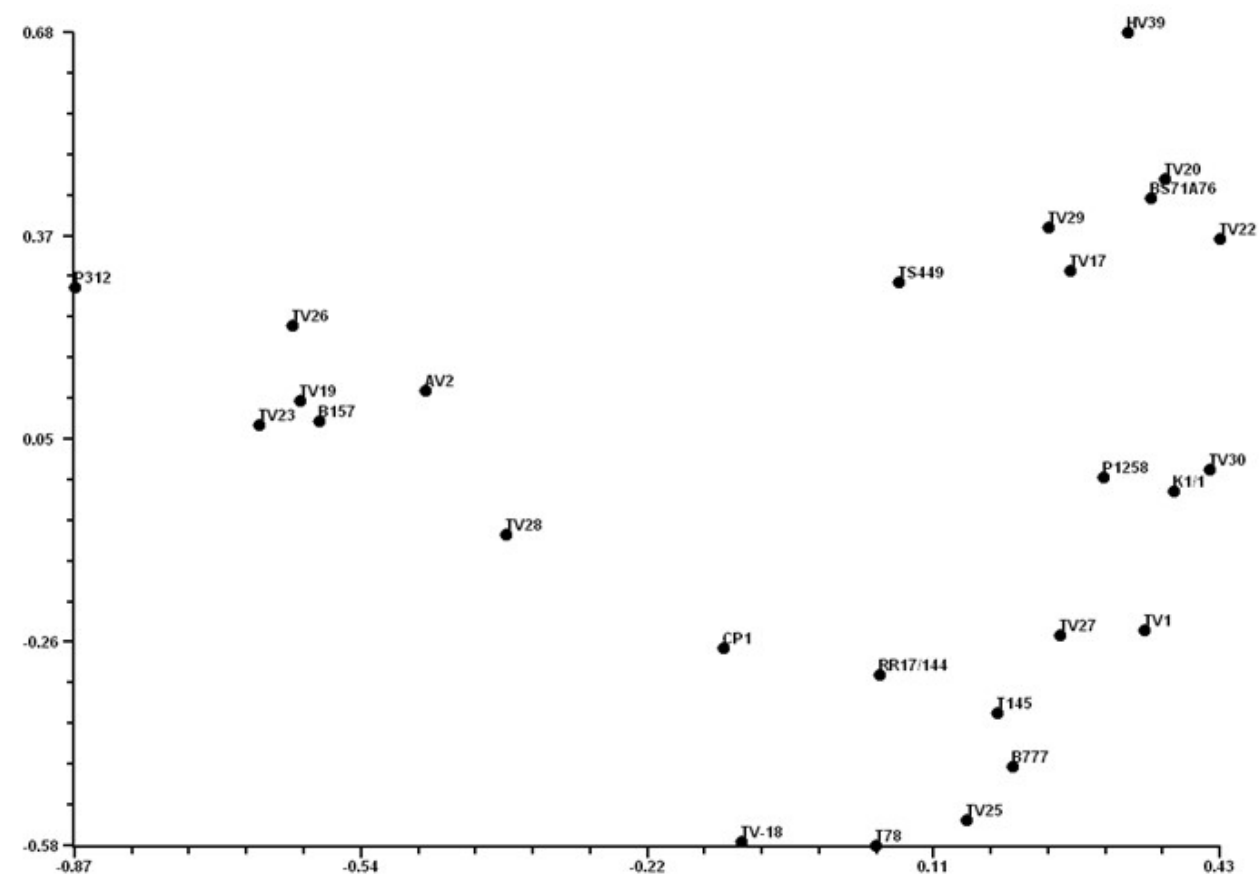

Figure 5. Correspondence analysis (2D plot) of twenty six tea cultivars

(Assam/China type), among this group K-1/1 revealed 100\% similarity with P-1258 and TV27 revealed $100 \%$ similarity with B777 again the pairs exhibited a lot of variation from the previous results TV30 and TV1 formed their own subgroups. Cluster II comprises of six cultivars: RR 17/144 (China hyb.), T145 (Assam hyb.), T78 (China hyb.), TV25 (Cambod), CP1 (Assam hyb.), TV18 (Cambod) with most of the Assam and cambod types and two 


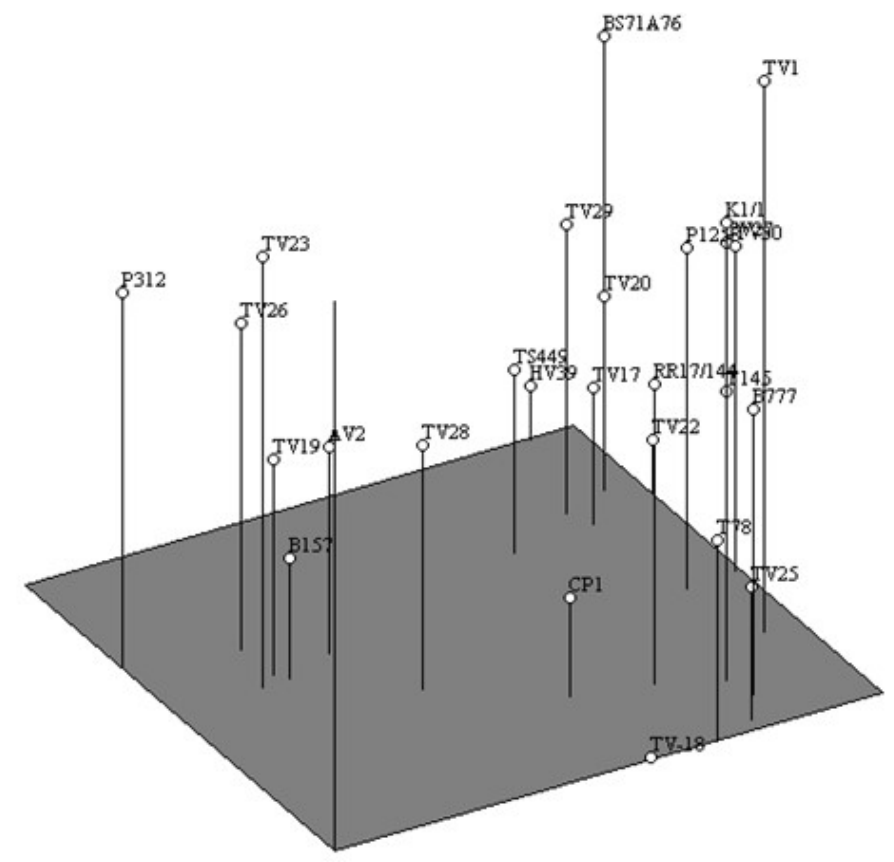

Figure 6. Correspondence analysis (3D plot) of twenty six tea cultivars

exceptions of China type. Cluster IIIA includes all Cambod cultivars: TV19, TV28, TV23, TV26 with a height similarity of $100 \%$ between TV28 and TV23 as well between TV19 and TV26.The second group of cluster IIIB comprises of all China cultivars AV2, B157, P312.

The same clusters were obtained in the dendrogram when the data was analyzed using NTSYSpc (Figure 4) and a 2D and 3D plot of correspondence analysis of the data was also in agreement with the dendrogram clustering (Figures $5 \& 6$ ) it also revealed three main clusters, Cluster I on the right of the plane includes Assam and Cambod varieties namely TV20, TV22, TV29, TV30, TV1, TV27, at a correlation coefficient of 0.18 , TV17 clustered very near to TV22 and TV20 which was also seen in the Dendrograms generated by UPGMA method. Cluster II in the middle includes Cambod varieties TV18 and CPI between a correlation coefficient of 0.27 and 0.18 with exceptions of RR 17/144, B777, T78. Cluster IIIA on left corner consists of all Cambod varieties namely TV19, TV26, TV28 and

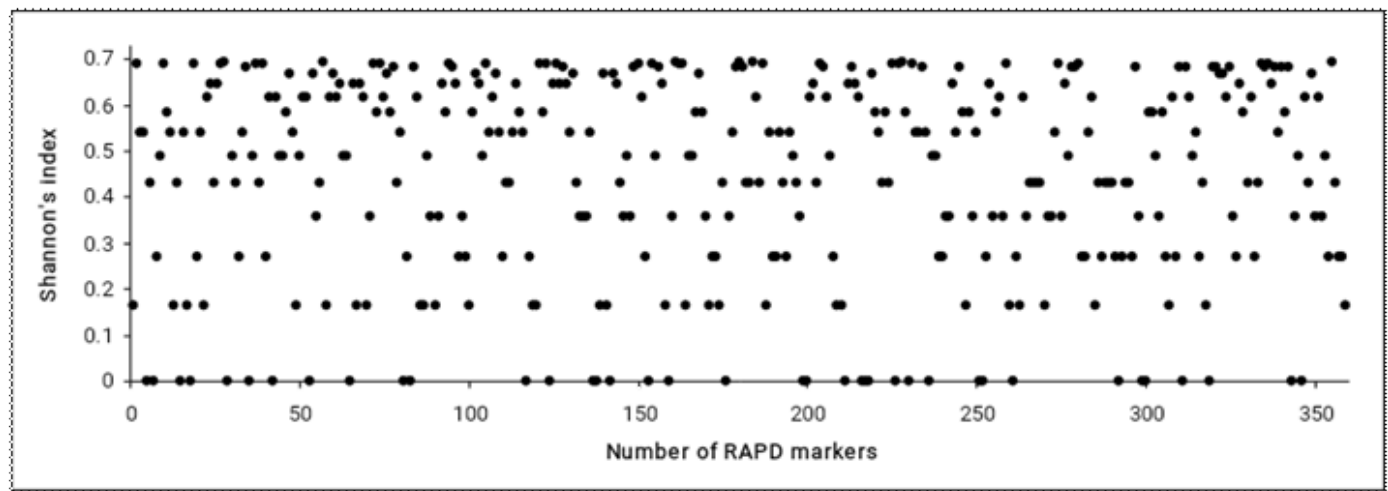

Figure 7. Shannon's index of diversity 
TV23. Cluster IIIB also on left comprises of China varieties most of which are Darjeeling clones namely P-312, B-157, AV2 between a correlation coefficient of 0.71 and 1.16 . One Chinese variety namely HV39 which is from Darjeeling and a TRA released variety clustered alone at the extreme right corner with a correlation coefficient of around 0.40.

Based on RAPD profile of each primer, measures of Shannon index of diversity were from 0.28 to 0.56 , with average of 0.44 (Figure7). This result shows that a relatively high level of polymorphism was determined by these random primers in the cultivars of tea.

\section{DISCUSSION}

The study of all twenty six cultivars within the Camellia sinensis using 34 decamer primers revealed their clear identification. This study demonstrates that RAPD offers a suitable means for the detection of genetic variability and molecular study of tea genotypes. Although the studies have been done on few varieties of tea found in India by various workers using RAPD technique but a complete study of twenty six varieties using 34 random deca-mer primers have been done for the first time in the present study revealing as high as $89.42 \%$ of polymorphism. Moreover, in this study molecular techniques like RAPD have been tagged with the statistical analysis using newly developed software's like POPGENE and NT-SYS which helped in characterization and identification of plant germplasm in a more improved way.

Selection of polymorphic alleles was carried out in a very careful manner and only clear and repeatable polymorphic bands were selected for the present study. 31 out of 34 primers could amplify all the samples and gave clear and repeatable banding patterns giving 321 polymorphic bands out of total of 359 bands. The percentage of polymorphism averaged at $89.42 \%$ when all the primers were considered together, whereas few primers also showed a $100 \%$ polymorphism among the varieties owing to their self incompatibility which makes them highly heterogeneous and consequently shows broad genetic variation among themselves.

Dendrograms constructed using both the software's POPGENE as well as NTSYSpc revealed the same result (Figs. $3 \& 4$ ) and is in complete agreement with the previous reports of tea taxonomy. Three main clusters have been formed and within these three clusters three types of tea can be easily differentiated Assam type, Cambod type and China type. The degree of polymorphism revealed in the present study was found to be $100 \%$ for some primers.

Correspondence analysis also revealed a clear picture of genetic variability as revealed by dendrograms constructed on the basis of both the software's. The Assam varieties clustered on right of the plane and the China types clustered on left and few Cambod cultivars clustered in the middle of the plane thus confirming their ancestral differences.

The mean genetic diversity and mean genetic distance among all the populations was 0.2893 and the genetic distance varied between 0.16 and 0.52 .

However, because the sample size and total amount of bands could influence the percentage of polymorphic bands value, other parameters that based on the frequency of polymorphic bands, such as Shannon's index of diversity and Simpson's index of diversity, are more suitable in estimating genetic variability (Cruzan 1998). The average of Shannon's index of diversity in the cultivars as studied is 0.44 (Table 3 ) indicating a relatively high degree of diversity among the tea population. The result is in agreement with the high percentage of polymorphism as detected by Nei's genetic diversity index. 
Therefore, it can be concluded from the present study that RAPD can be considered as a quick and effective means to establish the genetic relationships between the varieties without any prior knowledge of genomes or the use of polyacrylamide gels and radioactivity for resolution. Different cultivars can be conserved on the basis of their molecular differences under the light of the statistical analysis using newly developed softwares like POPGENE and NTSYSpc. The six primers OPA-10, OPB-04, OPB-05, OPB-9, OPB-12 and OPB-13 revealing $100 \%$ polymorphism may prove to be the most efficient markers obtained from this study along with OPA-04, OPA-11 and OPB-02 primers revealing as high as $87.5 \%$ polymorphism. These markers may provide a cheap, rapid and effective means to evaluate the genetic diversity among a large number of tea varieties and help devise sampling strategies to compliment classical morpho-agronomic descriptors and conservation of Indian tea cultivars.

\section{Acknowledgements}

The authors gratefully acknowledges the help of Dr. S.E. Kabir for providing the germplasm. This work was partially supported by a fast track young scientist project (No. SR/FTP/LS111/2000) awarded to A.S.

\section{LITERATURE CITED}

Banerjee, B. 1992. Botanical classification of tea, pp. 25-51. In: Wilson K.C. \& Clifford M.N. (eds.), Tea: cultivation to consumption, Chapman and Hall, London.

Bera, B. \& Saikia, H. 1999. Molecular characterization of tea (Camellia sinensis (L.) O. Kuntze) cultivars using DNA markers. Two and a bud. 46: $16-19$.

Cruzan, M.B. 1998. Genetic markers in plant evolutionary ecology. Ecology 79: 400 - 412.

Das, A.P. \& Ghosh, C. 2016. Camellia sinensis var. lasiocalyx (Watt) A.P. Das \& C. Ghosh - new combination for the Cambod variety of tea. Pleione 10(1): $167-168$.

De Bustos, A.; Cassanova, A.C.; Soler, C. \& Nouve, N. 1998. RAPD variation in wild populations of four species of the genus Hordeum. Theor. Appl. Gent. 96: $101-111$.

Dos Santos, J.B.; Nienhuis, J.; Skroch, P.; Tivang, J. \& Slocum, M.K. 1994. Comparison of RAPD and RFLP genetic markers in determining genetic similarity among Brassica oleracea L. genotypes. Theor. Appl. Genet. 87: 909 - 915.

Dellaporta, S.L.; Wood, J. \& Hicks, J.B. 1983. A plant DNA mini-preparation: version II. Plant Mol. Biol. Rep. 1: $19-21$.

Ghosh, H. 2001. Advances in selection and breeding of tea-a review. J. Plantation Crops 29: $1-17$.

Green, M.J. 1971. An evaluation of some criteria used in selecting large yielding tea clones. J. Agric. Sci. 76: 43 - 156.

Kaundan, S. \& Matsumoto, S. 2003. Identification of Processed Japanese Green Tea Based on polymorphisms generated by STS-RFLP Analysis. J. Agri. Food Chem.51: 1765 - 1770.

Kaundan, S.\& Park, Y. 2002. Genetic structure of six Korean tea populations as revealed by RAPD-PCR markers. Crop Sci. 42: 594 - 601.

Lerceteau, E.; Robert, T.; Petiard, V. \& Crouzillat, D. 1997. Evaluation of the extent of genetic variability among Theobroma cacao accessions using RAPD and RFLP markers. Theor. Appl. Genet. 95: 10 - 19.

Nei, M. \& Li, W.H. 1979. Mathematical model for studying genetic variation in terms of restriction endonucleases. Proc. Natl. Acad. Sci., USA,74: 5267 - 5273. 
Nei, M. 1972. Genetic distance between populations. Amer. Naturalist, 106: 283 - 292.

Paul, S.; Wachira, F.N.; Powell, W. \& Waugh, R. 1997. Diversity and genetic differentiation among populations of Indian and Kenyan tea (C. sinensis (L.) O. Kuntze) revealed by AFLP markers. Theor. Appl. Gent.94: 255-263

Powell, W.; Orozco, C.; Chalmers, K.J. \& Waugh, R. 1995. Polymerase chain reaction based assays for the characterization of plant genetic resources. Electrophoresis. 16: $1726-1730$

Prenner, G.A.; Bush, A.; Wise, R.; Kim, W.; Dommier, L.; Kasha, K.; Laroche, A.; Scoles, G.; Molner, S.J. \& Fedak, G. 1993. Reproducibility of random amplified polymorphic DNA (RAPD) analysis among laboratories. PCR Methods Applic. 2: 341 - 345.

Rohlf, F.J. 1998. Numerical Taxonomy and multivariate Analysis System, Version 2.0. Exeter Software, New York.

Smulders, M.J.M.; Bredemeijer, G.; Rus-Kortekass, W.; Arens, P. \& Vosman, B. 1997. Use of short microsatellites from data base sequences to generate polymorphisms among Lycopersicon esculentum cultivars and accessions of other Lycopersicon species. Theor. Appl. Genet. 97: $264-272$.

Sun, G.L.; Salomon, B.; Bothermer, R.V. 1998. Characterisation and analysis of microsatellite loci in Elymus caninus (Triticeae: Poaceae). Theor. Appl. Genet. 96: 676-682.

Vos, P.; Hogers, R.; Reijans, M.; Van Der Lee, T.; Hornes, M.; Frijters, A.; Pot, J.; Peleman, J.; Kuiper, M. \& Zabeau, M. 1995. AFLP: A new technique for DNA fingerprinting. Nucleic Acids Res. 23: 4407 - 4414.

Wachira, F.N'; Waugh, R.; Hackett; C.A. \& Powell, W.1995. Detection of genetic diversity in tea (Camellia sinensis) using RAPD markers. Genome 38:201 - 210.

Wight, W. 1962. Tea classification revised. Curr. Sci. 31: 298 - 299.

Wilkremaratne, M.R. 1981. Variation in some leaf characteristics in tea (Camellia sinensis) and their use in identification of clones. Tea Quart. 50: 183 - 189.

Williams, J.G.K.; Kubelik, A.R.\& Tingey, S.V. 1990. DNA Polymorphisms amplified by arbitrary primers are useful as genetic markers. Nucleic Acids Res. 18: 6531 - 6535.

Xu, R.Q.; Tomooka, N.; Vaughan, D.A. 2000. AFLP markers for characterizing the Azuki bean complex. Crop Sci. 40: 808 - 815 .

Yeh, F.C.; Young, R.C.; Timothy, B.; Boyle, T.B.J.; Ye, Z.H. \& Mao, J.X. 1997. POPGENE, the user friendly shareware for population genetics analysis. Molecular Biology and Biotechnology Center, University of Alberta, Canada. (http://www.ualberta.ca/ fyeh; verified September 27, 2001).

Zhang, Q.; Saghai Maroof, M.A.; Lu, T.Y. \& Shen, B.Z. 1992. Genetic diversity and differentiation of indica and japonica rice detected by RFLP analysis. Theor. Appl.Genet. 83: 495-499.

Zhang, Q.; Saghai Maroof, M.A.; Lu, T.Y. \& Shen, B.Z. 1992. Genetic diversity and differentiation of indica and japonica rice detected by RFLP analysis. Theor. Appl.Genet. 83: 495-499. 